\title{
Competition of Crystalline and Liquid Crystalline Moieties in Self-assembly of Poly(oxyethylene) Cholesterol Ethers
}

\author{
Jun-Ting Xu*, Liang Xue, Zhi-Qiang Fan
}

Department of Polymer Science \& Engineering, Zhejiang University, Hangzhou 310027, China

\author{
Zhong-Hua $\mathrm{Wu}$
}

Beijing Synchrotron Radiation Laboratory, Institute of High Energy Physics, Chinese Academy of Sciences, Beijing 100039, China

\section{Jin Kon Kim}

National Creative Research Center for Block Copolymer Self-Assembly, Department of Chemical Engineering and Polymer Institute, Electronic and Computer Engineering Division, Pohang University of Science and Technology, Pohang, Kyungbuk 790-784, Korea 
Figure S1 shows GPC traces for all ChEOn $(n=5 \sim 45)$. The polydispersity index of ChEOn with $\mathrm{n} \geq 15$ is 1.11 , whereas those for ChEO5 and ChEO10 are 1.20 and 1.22, respectively.

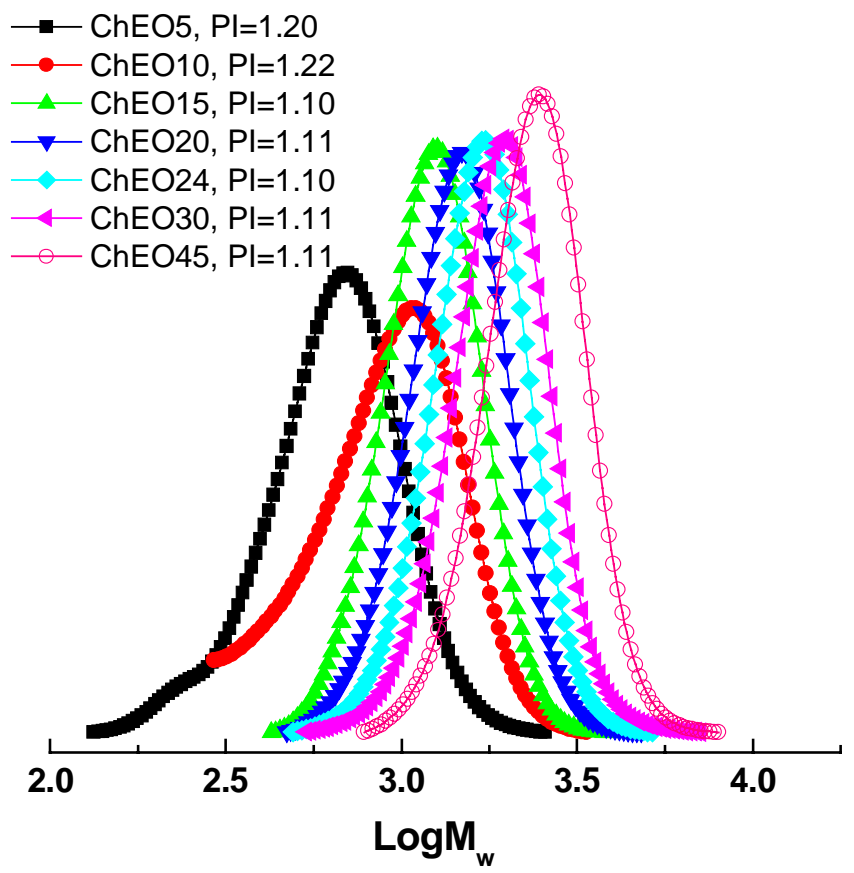

Figure S1. GPC curves of ChEOns. 
Figure S2(a) S2(f) give ${ }^{1} \mathrm{H}-\mathrm{NMR}$ spectra for all ChEOn $(\mathrm{n}=5 \sim 45)$. The assignments of nmr peaks are as follows.

(1) $5.34 \mathrm{ppm}(1 \mathrm{H}$, unsaturated proton in cholesterol moiety); (2) 3.5-3.8 ppm (4×n H, protons in POE moiety); (3) $3.18 \mathrm{ppm}(1 \mathrm{H}$, proton in cholesterol bonded to POE moiety); (4) 2.5-3.0 ppm (1H, broad peak, proton in hydroxyl end group of POE moiety); and (5) 0.67-2.5 ppm (43H, aliphatic protons in cholesterol moiety).

The mole fraction of POE moiety in ChEOn was obtained by the ratio of the peak at 3.5-3.8 ppm corresponding to the protons of POE moiety except the end hydroxyl group to that at $0.6 \mathrm{ppm}$ to $2.5 \mathrm{ppm}$ corresponding to the aliphatic protons in cholesterol moiety. These measured values, as shown in Table S1, are within $5 \%$ error compared with the values calculated from chemical structure shown in Figure 1 at a given $\mathrm{n}$.

Table S1. Experimentally measured and stoichiometrically calculated mole fractions of POE moiety in ChEOn

\begin{tabular}{|l|l|l|}
\hline \multirow{2}{*}{ Samples } & \multicolumn{2}{|c|}{$\mathrm{I}_{3.5-3.8 \mathrm{ppm}} / \mathrm{I}_{0.6-2.5 p m ~ a)}$} \\
& Measured & Calculated \\
\hline ChEO5 & 0.47 & 0.49 \\
\hline ChEO10 & 0.93 & 0.95 \\
\hline ChEO15 & 1.40 & 1.42 \\
\hline ChEO20 & 1.86 & 1.89 \\
\hline ChEO24 & 2.23 & 2.23 \\
\hline ChEO30 & 2.79 & 2.82 \\
\hline ChEO45 & 4.18 & 4.23 \\
\hline
\end{tabular}



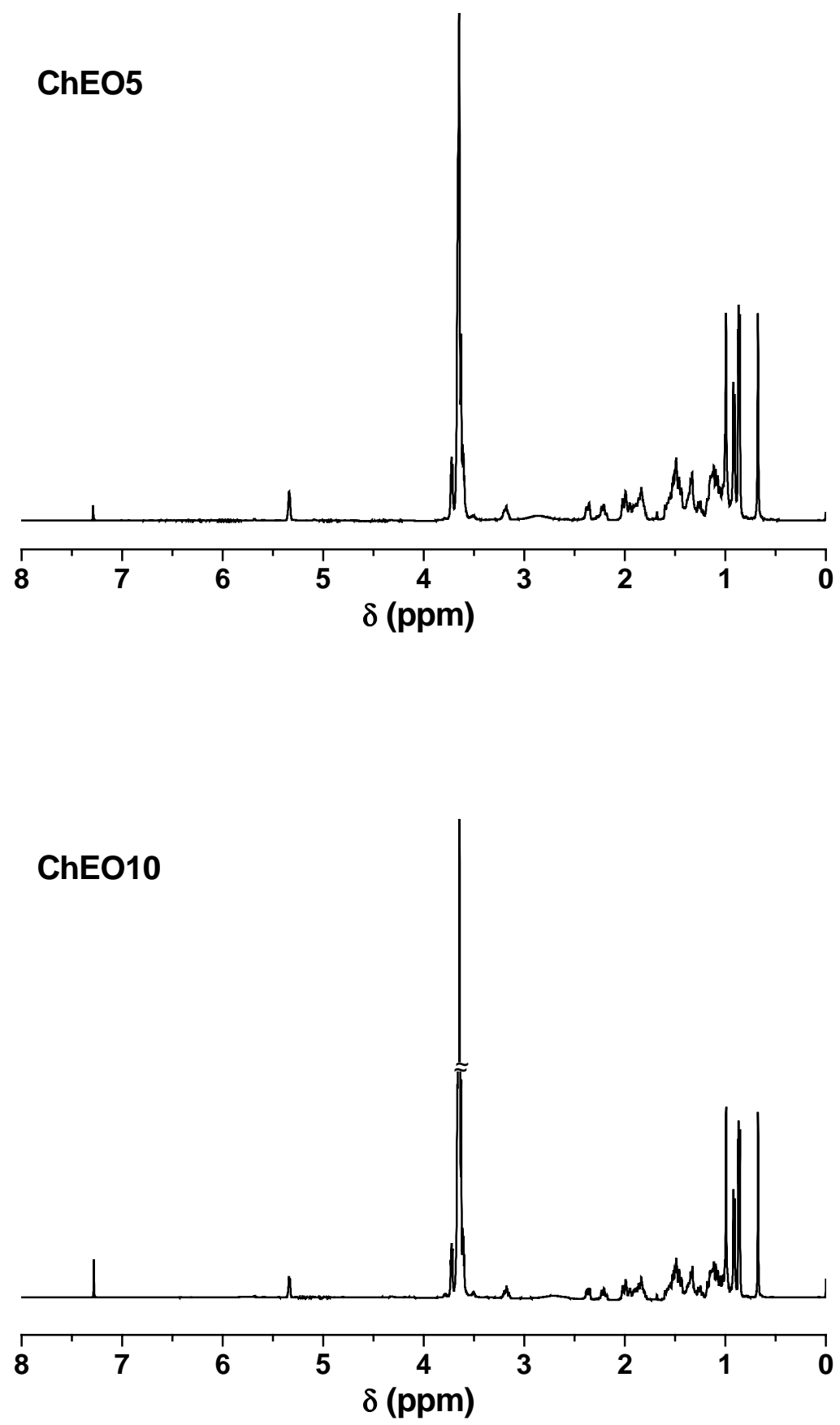

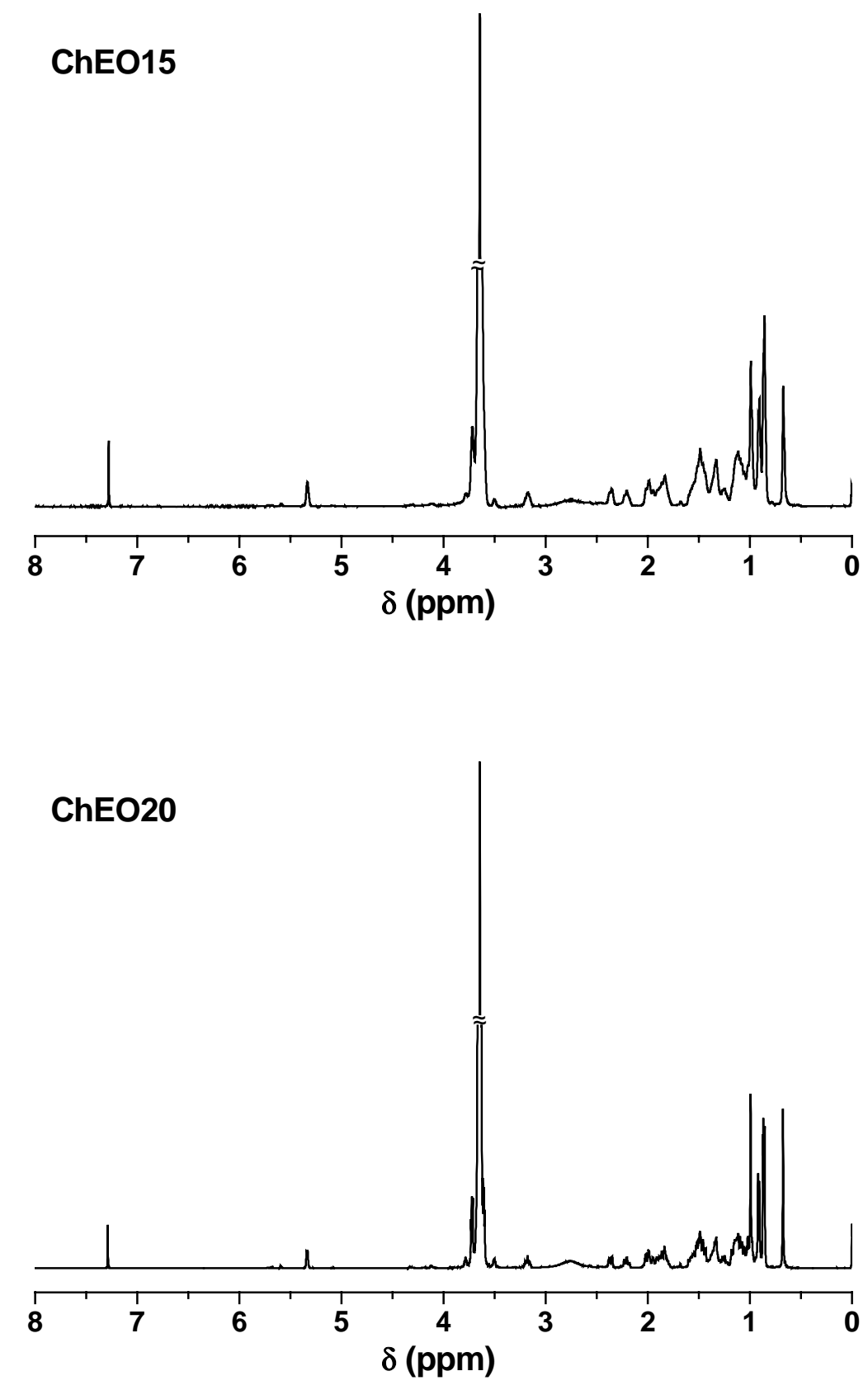

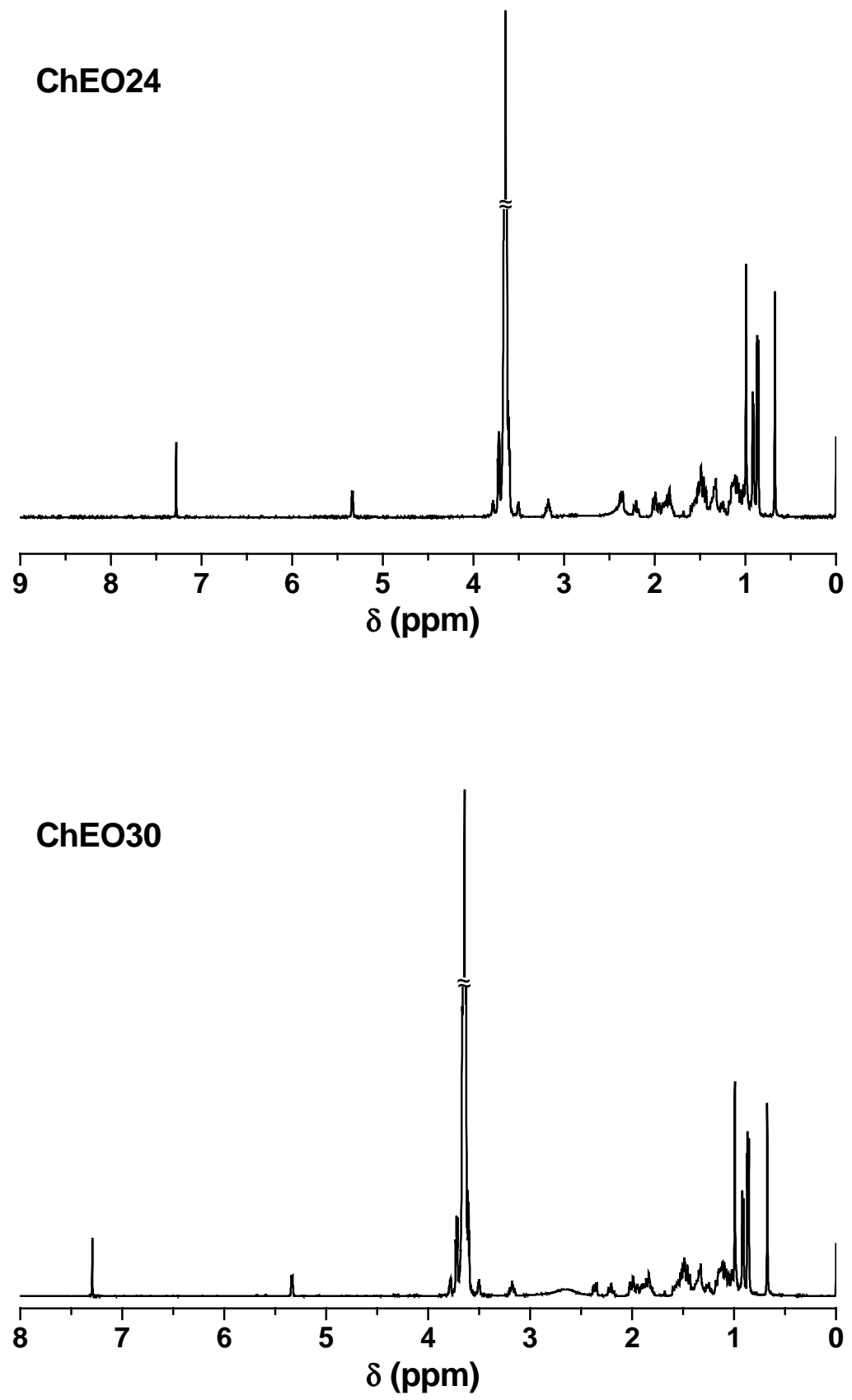

Figure S2. ${ }^{1} \mathrm{H}-\mathrm{NMR}$ spectra of all ChEOns. 
Figure S3 shows the 2D SAXS images of ChEO10 and ChEO15.
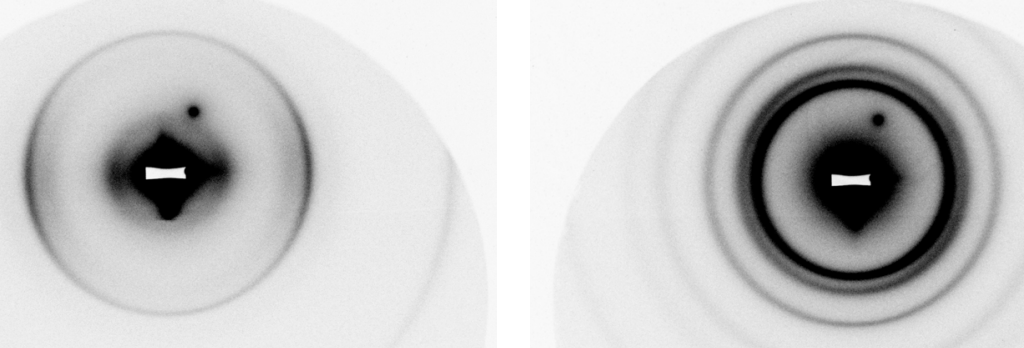

(a)

(b)

Figure S3 2D SAXS images of ChEO10 (a) and ChEO15 (b). 
Figure S4 gives (a) the length and (b) the width for one cholesterol molecule, which are calculated from the software of "ChemOffice".
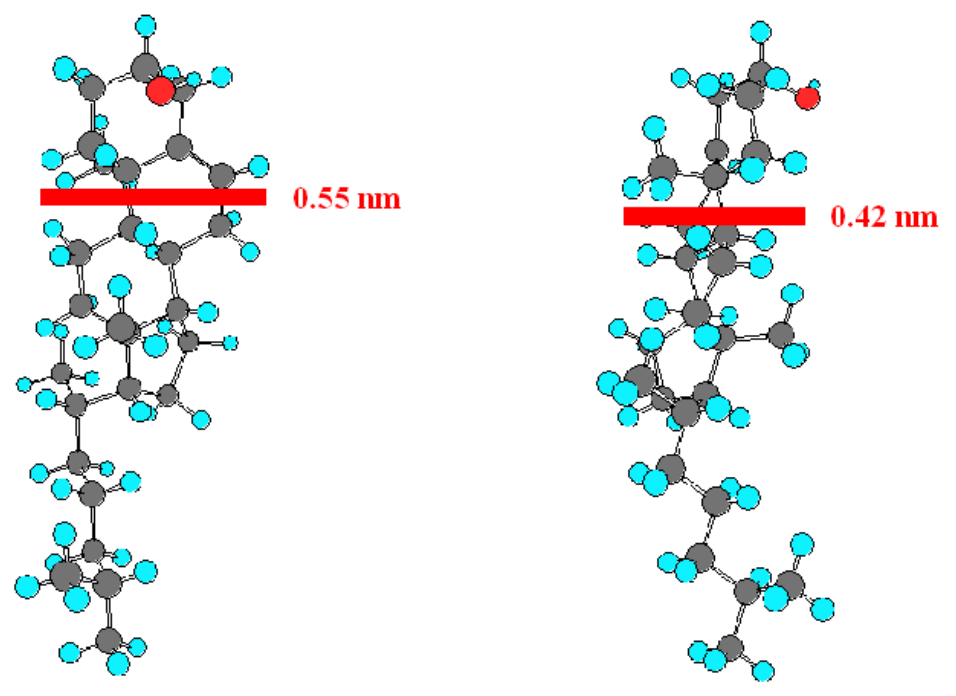